\title{
Prosthodontic Complications Associated with Immediately Loaded Fixed Implant-Supported Prostheses - A Review
}

\author{
Tanvi Rajesh Balwani ${ }^{1}$, Surekha Dubey Godbole², Seema Sathe ${ }^{3}$, Aditi Vinay Chandak ${ }^{4}$, Shanvi Agrawal ${ }^{5}$ \\ 1, 2, 3, 4, 5 Department of Prosthodontics, Sharad Pawar Dental College, Sawangi, Maharashtra, India.
}

\section{ABSTRACT}

Immediate loading and placement of dental implants with full-arch fixed implantsupported prostheses has been stated as a very successful treatment modality. Definitely it's becoming a trending option as it provides patient services by reforming transition from removable to fixed prosthesis. It ultimately eliminates the requirement for an interim denture. Thus, it reduces the time required in the process. However, attaining and retaining a successful treatment result will have to face its own challenges. From a prosthodontic point of view, there are a number of complications which can occur in all the phases involved in the treatment process. This review article describes the most commonly occurring prosthetic complications that are associated with full-arch fixed implant-supported prostheses. Treatment of the edentulous patients with implant-supported restorations presents a significant challenge to the treating clinician. Patient expectations with regard to aesthetics, phonetics, form and function are high. There is a myriad of factors that needs to be evaluated to determine if the patient is a suitable candidate for a fixed vs. a removable implant-supported restoration. Evaluation of the edentulous patient is also complicated by the fact that patients may not only be missing clinical crown height but in addition may have experienced a combination of tooth, soft tissue and bone loss, with associated changes in facial form. Here in this article, various strategies to combat the complications have been discussed.

\section{KEY WORDS}

Immediate Loading, Dental Implants, Prosthetic Complications.

\section{Corresponding Author:} Dr. Tanvi Rajesh Balwani, Near Ramakrishna Hotel, Subhedar Layout. Jhulelal Colony, Wardha, Maharashtra, India.

E-mail: tbalwani16@gmail.com

DOI: $10.14260 /$ jemds/2020/745

How to Cite This Article:

Balwani TR, Godbole SD, Sathe S, et al. Prosthodontic complications associated with immediately loaded fixed implantsupported prostheses - a review. J Evolution Med Dent Sci 2020;9(45):3394-3398, DOI: 10.14260/jemds/2020/745

Submission 04-06-2020,

Peer Review 25-09-2020,

Acceptance 05-10-2020,

Published 09-11-2020.

Copyright (C) 2020 Tanvi Rajesh Balwani et al. This is an open access article distributed under Creative Commons Attribution License [Attribution 4.0 International (CC BY 4.0)] 


\section{BACKGROUND}

Before enumerating various prosthetic complications, first step is to know about the misconceptions about immediately placing and loading protocols of implants with complete arch fixed implant supported prosthesis.

It is a single procedure, most common misconception. It is not a single procedure rather it's a process as are other prosthodontics treatment options. All the five phases involved in the treatment have their own challenges. Those are the diagnostic phase, surgical procedure, transitional prosthodontic phase, definitive prosthodontics phase and maintenance phase. To accomplish successful outcome clinicians should identify, prevent and deal with the complications associated with all the phases.

Secondly this treatment process is exclusively a surgical technique. This is also a misconception. Although it has been popular due to the innovative utilization of tilted implants. Also, its ability to neglect grafting procedures, ${ }^{1}$ the fact still is that this treatment modality is related to prosthodontics with a strong surgical component. Surgical intervention plays a crucial role for the success of the treatment modality still eventually it also can lead to prosthesis complication. This will be put emphasis on in the further discussion.

Biologic and prosthetic complications are commonly exclusive. This is the third misconception. Biologic complications are defined as those that relate to dental implant osseointegration, bone-level changes and soft-tissue responses. $^{2}$

According to facts, a correlation is present between biologic and prosthetic aspects. For instance, soft tissue inflammation is the outcome of improperly contoured prosthesis. At last, finally amplification related to prosthetic complications is that materials used for the fabrication of prosthesis plays a crucial role. The most studied and accepted type of complete arch fixed implant-supported prosthesis is metal - acrylic prosthesis. ${ }^{3}$ Various materials such as such as zirconia and metal ceramic have been used. It has been stated that their expected results have been less frequent and have short follow up period. ${ }^{4}$

Metal-acrylic prostheses are very common. It remains the point of reference to which other prostheses are measured. So, it is going to be the focus.

\section{General Prosthetic Complications}

The easiest method to know all the prosthetic complications is made after review of findings got in complete treatment procedure. Up till now there have been 3 systematic reviews which have been published, $3,5,6$ that amalgamate the evidence of studies which have examined complications associated with complete-arch fixed implant-supported prostheses of metal acrylic.

Generally, they show the expected results with survival rates ranging from 93 to $100 \%$ and 82 to $100 \%$ after 5 years and 10 years respectively. ${ }^{3}$ Still there occurs a lot many prosthodontic complications. They are classified as being either structural, cosmetic or functional. ${ }^{7}$ Mechanical and technical aspects of components of implants are main emphasis of structural complications. And two factors which are patient specific are functional and aesthetic complication.
Structural complications are highest amongst the other complications as seen in the systematic reviews. Till now the commonest structural complication seen was fractured veneered acrylic. It has complication rate of $\approx 33 \%$ and $\approx 66$ $\%$ at 5 and 10 years respectively. ${ }^{6}$

Second commonly reported structural complication is of prosthetic screw loosening with 5-year complication rate of $\approx$ $10 \%$ and 10 year complication rate $\approx 20 \%$. There are numerous numbers of structural complications, in increasing order of incidence were fractured opposing restoration and the metal framework, prosthetic screw fracture, prosthesis wear and requirement for total substitute of acrylic resin teeth, and loss of access channel restoration. ${ }^{6}$

The least reported complication is of aesthetic and functional aspect. In history patients accounted with very great gratification with their prostheses. ${ }^{8}$ Studies reported that there is only $2 \%$ complication rate of this aspect. ${ }^{6}$

However, the more common functional complications reported is phonetics. ${ }^{9}$ Historically this was apparent when prosthetic driven implants were not a mainstream. Henceforward, implants were mostly placed beyond the boundaries of the prosthesis, which eventually lead to irregularities in the contours lingually. And thus, those are a smaller amount conductive to smooth articulations with the tongue.

Soft-tissue hyperplasia and inflammation inferior to the prosthesis is also a reported prosthesis related biological complications. Its complication rate was $=26 \%$ in 10 years.

\section{Prosthetic Complications in Various Phases of Treatment}

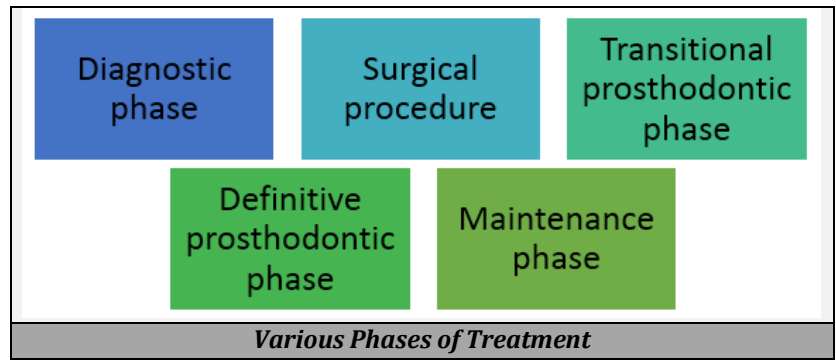

\section{The Diagnostic Phase}

This phase involves careful evaluation of patient and collection of detailed records which enables the key factors that will ultimately reflect in the final prosthetic prosthesis result. Complications caused in diagnostic phase have unknown aetiology. From mechanical or aesthetic point of view it may eventually lead to suboptimal prosthesis. They usually occur due to failure in recognition of the features stated below.

1.1 Occlusal Vertical Dimensions - As already stated in the systematic reviews, the most common material used is acrylic / resin for fixed implant-supported prostheses. The disadvantage of this material is that it is weak and strength is achieved with bulk. Due to which minimum of 12 to $15 \mathrm{~mm}$ of restorative space is required in each arch. Or else, fracture rate of the prosthesis will be higher. So optimum amount of restorative space is required in such cases which can be achieved by 2 ways: Firstly, with the 
help of surgical reduction of the alveolar ridge or else increase in OVD. In numerous occurrences, a combination of both ways can also be carried out. But every time it's not possible. As a result, a knowing of the patient's OVD is essential. When there is loss of occlusal vertical dimension there is need to achieve restorative space by increase in the OVD. Reducing the alveolar ridge is the only method to gain restorative space left when there is no loss in the OVD. Usually it is not always possible. ${ }^{10,11}$

1.2 Smile Line Related to Transition Zone - The transition zone is the prosthesis-gingival junction. In situations when the transition zone is exposed there is need for more alveolar ridge reduction which must be carried out at the time of surgery. This is also applicable when sufficient restorative space is present for fixed prosthesis. Or else the contrast from synthetic pink acrylic and natural pink gingiva will be obvious which will eventually lead to high suboptimal aesthetics. The practitioner should always assess the smile line with a non-posed smile. A number of patients opting for complete arch fixed prosthesis usually do not smile so often because they are very embarrassed. After the treatment is completed this scenario will arise because then the patients would smile more often. 12

1.3 Lip Support - In cases where the patient had been on removable prosthesis for a longer period of time, they often require flange buccally for adequate lip support for pleasing aesthetics. The buccal flange cannot have fixed prostheses as the concave intaglio surface accumulates plaque and food debris hence it would prevent accessibility for oral hygiene. This problem is usually encountered when only less amount of vertical residual resorption is present. In these cases, the emergence profile is needed to be responsible for the required lip support which would be steep. So, to avoid these circumstances, it is necessary for planning significant amount of vertical ridge reduction that permits a gradual emergence profile which will provide lip support.

1.4 Centric Relation - Amongst all diagnostic features the one which plays less crucial role compared to rest of the features is centric relation. Mostly patients who wants treatment for complete arch rehabilitation usually because of the broken dentition develop a modified jaw position so that they can masticate food. And so, to record the jaw relation of patient with the given inaccurate results in transitional fixed prosthesis that would not occlude which lead to uneven distribution of forces. So, consequence which is faced is fractured interim prosthesis. Although, this is an aspect that can be rectified from transitional to the definitive phase. With the aim of avoiding such circumstances, clinicians must keep interocclusal records in centric relation so that the predictable position is available.

\section{The Surgical Procedure}

This review article has not focused on the surgical protocols for placement of immediate implants but yet many other factors are also there which should be highlighted about the surgical procedure which would directly affect the prosthetic prosthesis outcome. As likely as seen in the diagnostic phase the complication arising from it have unknown aetiology and are irreversible.
Whenever certain precautionary measures are taken into account the actual procedure carried out surgically is simplified. ${ }^{13}$ Nevertheless, it becomes the surgeon's responsibility to precisely carry on with prosthodontics treatment plan. This predominantly requires sufficient amount of alveolar ridge reduction for creating sufficient restorative space for prosthetic materials.

Next is surgeon must keep in mind that dental implants are placed within the boundaries of bone and confines of prosthesis. This can definitely be avoided by the use of surgical stent as well as experience of the surgeon. ${ }^{14}$

Consequently, with surgeon's negligence discomfort will be faced by patient from the prosthesis. Eventually which becomes an irreversible complication which couldn't be solved without repositioning the implants. Nowadays guided surgical technique even if not used, planning done digitally can help in providing proposed positions of the implants.

\section{The Transitional Prosthodontic Phase}

It mainly starts immediately after the implant loading with interim prosthesis. And this phase overlaps with the definitive phase. Acrylic material is used for fabrication of interim prosthesis. Henceforth, as the prosthesis is inherently weaker it's more susceptible to fracture. As stated in the systematic reviews mention previously in the article this is most reported prosthetic complication..$^{5}$ By chance, acrylic can be repaired very often with the support of laboratory. It is also feasible.

The patients suffering from parafunctional habits or had fractured multiple restorations, precautionary measures should be there, in order to reduce the chances of fracture and simply future repairs.

These includes the reduction or eradication of all cantilevered portions of the prosthesis, ${ }^{15}$ incorporating the metal wire reinforcement and verifying that the occlusion is evenly distributed amongst all the prosthetic teeth.

As the transitional phase is a bright chance to rectify and correct the problems encountered in the first 2 phases which eventually allows patient to accustomed to the prosthesis and as well as prevent fracture. At last, this plays a crucial role for the clinician in making decisions regarding the changes which will be required for the final prosthesis.

\section{The Definitive Prosthodontic Phase}

The phase is the most important one as final design and fabrication process is carried out in this phase. If possible, adjustments whichever are needed should be done in the interim restorations. Due to which there are minimal steps required in designing. As already modification is carried out in interim restoration and due to which complication, that could have occurred in definitive phase can be avoided. The most crucial aspect of this phase is it should be kept in mind that definitive prosthesis framework maintains whole passivity on the implants. ${ }^{16}$

Or else, there would be unnecessary straining on the prosthetic screws which helps to retain the prosthesis. Inadequate passive fit has been the main reason for screw loosening and eventual fractured screw. ${ }^{6}$ During recording the impression the easiest way to confirm accuracy in capturing position of the implants is by splitting the impression copings.

Even though it's uncommon it plays a role in recording fixture level impressions. ${ }^{17}$ In addition, the frameworks which 
are milled have achieved greater amount of passive fit as compared with conventional cast frameworks. ${ }^{2}$

Certainly, in accordance with prosthetic teeth, every required precautionary measure should be made in order to evenly distribute the occlusal contacts bilaterally and limit the amount of tooth wear. The usage of T-scan has been recently now become a trending option as it helps clinicians to verify and adjust the uneven occlusal contacts and forces. Highly cross - linked acrylic teeth should be used to resist wear. ${ }^{10}$ Practitioner's should put their effort to deliver mutually protected articulation in order to neglect deep vertical overlap with small horizontal overlap. ${ }^{18}$ Patients should therefore have freedom of movement to move to and from centric relation. Whenever, guidelines are avoided, the patients more often experience prosthetic fractures.

As far as the contours of the prosthesis are considered, the intaglio surface has to be made flat to convex, not festooned and closely abutted to the tissues. ${ }^{19,20}$ Buccolingually the profile needs to be smooth which would help in phonation. Or else, patient may face phonetic challenges.

Finally, and the most significantly, it is paramount that the final restoration is designed such that screw retention remains the major factor.

\section{The Maintenance Phase}

The last but the most important phase is the maintenance phase. As soon as the definitive phase is completed that is definitive prosthesis insertion has been done, this phase begins and continues indefinitely. At present, prosthetic complications are merely an eventuality. Not like some of the other phases of treatment, they don't have unknown aetiology. The most important aspect of immediate loading protocol is that as soon as the treatment is done the patients will have interim prosthesis which is added advantage of this protocol. Although the repair cannot be accomplished on the same but still the patient can manage with provisional restoration till the time repairs are being done.

In addition, it is compulsory to monitor the occlusion by providing frequent recall on regular intervals. It is helpful to have frequent recall to monitor the integrity of posterior occlusion. It determines the need for a refurbishing of the prosthetic teeth According to a retrospective chart review, from the $2^{\text {nd }}$ to 5 th year of post-insertion, there is 50 times probability that patients will require replacement of prosthetic teeth. ${ }^{21}$ Moreover, these recall visits allows the practitioner to test the efficacy of implants, observe the maintenance. Note the soft tissues changes, as well as modifications required in contours of the prosthesis as required. ${ }^{22}$

\section{CONCLUSIONS}

Complete overall planning and execution is required for the success of immediate loaded complete arch fixed implantsupported prostheses. There can be numerous complications with this treatment modality but all those can be handled skillfully by treatment planning and avoiding the complications arising with it. This requires considerable amount of efforts by the practitioner so as to deal with the prosthetic complications. It requires immense skills for planning challenges associated with the treatment process.

Financial or other competing interests: None.

Disclosure forms provided by the authors are available with the full text of this article at jemds.com.

\section{REFERENCES}

[1] Maló P, Rangert B, Nobre M. All-on-4 immediate-function concept with Brånemark System implants for completely edentulous maxillae: a 1-year retrospective clinical study. Clin Implant Dent Relat Res 2005;7 Suppl 1:S88-94.

[2] Drago C, Howell K. Concepts for designing and fabricating metal implant frameworks for hybrid implant prostheses. J Prosthodont 2012;21(5):413-24.

[3] Kwon T, Bain PA, Levin L. Systematic review of short-(510 years) and long-term (10 years or more) survival and success of full-arch fixed dental hybrid prostheses and supporting implants. J Dent 2014;42(10):1228-41.

[4] Abdulmajeed AA, Lim KG, Närhi TO, et al. Complete-arch implant-supported monolithic zirconia fixed dental prostheses: a systematic review. J Prosthet Dent 2016;115(6):672-7.e1.

[5] Menini M, Signori A, Tealdo T, et al. Tilted implants in the immediate loading rehabilitation of the maxilla: a systematic review. J Dent Res 2012;91(9):821-7.

[6] Papaspyridakos P, Chen CJ, Chuang SK, et al. A systematic review of biologic and technical complications with fixed implant rehabilitations for edentulous patients. Int J Oral Maxillofac Implants 2012;27(1):102-10.

[7] Schmitt A, Zarb GA. The longitudinal clinical effectiveness of osseointegrated dental implants for single-tooth replacement. Int J Prosthodont 1993;6(2):197-202.

[8] De Bruyn H, Collaert B, Lindén U, et al. Patient's opinion and treatment outcome of fixed rehabilitation on Brinemark implants. A 3-year follow-up study in private dental practices. Clin Oral Implants Res 1997;8(4):26571.

[9] Sadowsky SJ. The implant-supported prosthesis for the edentulous arch: design considerations. J Prosthet Dent 1997;78(1):28-33.

[10] Sadowsky SJ, Fitzpatrick B, Curtis DA. Evidence-based criteria for differential treatment planning of implant restorations for the maxillary edentulous patient. J Prosthodont 2015;24(6):433-46.

[11] Sadowsky SJ, Hansen PW. Evidence-based criteria for differential treatment planning of implant restorations for the mandibular edentulous patient. J Prosthodont 2014;23(2):104-11.

[12] Goodacre CJ, Bernal G, Rungcharassaeng K, et al. Clinical complications with implants and implant prostheses. J Prosthet Dent 2003;90(2):121-32.

[13] Da Cunha MC, Santos JFF, Santos MBF, et al. Patients' expectation before and satisfaction after full-arch fixed implant-prosthesis rehabilitation. J Oral Implantol 2015;41(3):235-9.

[14] The glossary of prosthodontic terms. J Prosthet Dent 2005;94(1):10-92.

[15] Drago C. Cantilever lengths and anterior-posterior spreads of interim, acrylic resin, full-arch screw-retained 
prostheses and their relationship to prosthetic complications. J Prosthodont 2017;26(6):502-7.

[16] Spazzin AO, Camargo B, Bacchi A. Ensuring passivity and retrievability for immediate complete-arch implantsupported prostheses. J Prosthet Dent 2017;117(2):2147.

[17] Papaspyridakos P, Hirayama H, Chen CJ, et al. Full-arch implant fixed prostheses: a comparative study on the effect of connection type and impression technique on accuracy of fit. Clin Oral Implants Res 2016;27(9):1099105.

[18] Davies SJ, Gray RJM, Young MPJ. Good occlusal practice in the provision of implant borne prostheses. Br Dent J 2002;192(2):79-88.
[19] Schnitman P. The profile prosthesis: an aesthetic fixed implant-supported restoration for the resorbed maxilla. Pract periodontics Aesthet Dent 1998;11(1):143-51.

[20] Del Castillo R, Ercoli C, Delgado JC, et al. An alternative multiple pontic design for a fixed implant-supported prosthesis. J Prosthet Dent 2011;106(3):198-203.

[21] Purcell BA, McGlumphy EA, Holloway JA, et al. Prosthetic complications in mandibular metal-resin implant-fixed complete dental prostheses: a 5-to 9-year analysis. Int J Oral Maxillofac Implants 2008;23(5):847-57.

[22] Nimonkar S, Belkhode VM, Sathe S, et al. Prosthetic rehabilitation for hemimaxillectomy. J Datta Meghe Inst Med Sci Univ 2019;14(2):99-102. 